
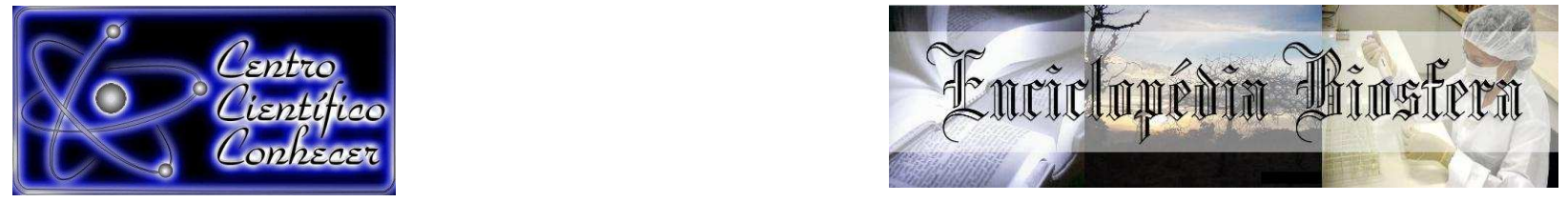

\title{
CARACTERÍSTICAS DA TERMORREGULAÇÃO ANTES E APÓS DIFERENTES TEMPOS DE EXPOSIÇÃO AO CALOR EM FRANGOS DE CORTE
}

\footnotetext{
Mara Regina Bueno de Mattos Nascimento ${ }^{1}$, João Paulo Rodrigues Bueno ${ }^{2}$, Otávio Cintra Lemos Olivieri ${ }^{3}$, Rodrigo Lemos Olivieri Rodrigues Alves ${ }^{3}$, Fernanda Marcondes de Rezende ${ }^{4}$

${ }^{1}$ Professora Doutora da Faculdade de Medicina Veterinária da Universidade Federal de Uberlândia. Uberlândia, Minas Gerais, Brasil, maran@umuarama.ufu.br

${ }^{2}$ Doutorando em Ciências Veterinárias pela Universidade Federal de Uberlândia.

${ }^{3}$ Graduandos em Medicina Veterinária pela Universidade Federal de Uberlândia.

${ }^{4}$ Professora Doutora da Faculdade de Medicina Veterinária da Universidade Federal de Uberlândia.
}

\section{Recebido em: 08/09/2015 - Aprovado em: 14/11/2015 - Publicado em: 01/12/2015 DOI: http://dx.doi.org/10.18677/Enciclopedia_Biosfera_2015_106}

\begin{abstract}
RESUMO
Objetivou-se investigar o efeito do estresse por calor após uma, duas ou três horas em características termorreguladoras de frangos de corte avaliadas aos 41 dias de idade. Foram alojados 560 pintos de corte, machos, da linhagem Cobb ${ }^{\circledR}$, distribuídos em 16 boxes com 35 aves em cada boxe. Do primeiro ao $13^{\circ}$ dia de idade foram

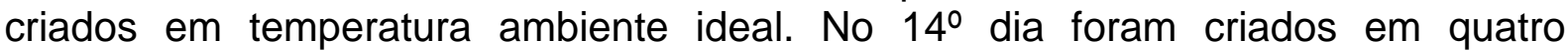
ambientes térmicos sendo um em conforto térmico e os outros três ambientes sob temperatura ambiente elevada por uma, duas e três horas, respectivamente. Aos 41 dias de idade antes do estresse pelo calor e depois foram mensurados as temperaturas da crista, cabeça, asa, dorso e pata e a temperatura cloacal e calculou-se a temperatura superficial média (TSM). No momento das avaliações mediram-se as temperaturas do ar e do globo, umidade e velocidade do ar. Foram calculados a média e o desvio-padrão das medidas de termorregulação para os quatro ambientes térmicos nos períodos antes e após estresse térmico. $\mathrm{O}$ teste $\mathrm{t}$ não-pareado foi utilizado para comparar a média do grupo antes com o grupo depois do estresse por calor em cada ambiente térmico. As temperaturas cloacal e superficiais, após exposição ao calor nos três ambientes térmicos (1h, $2 \mathrm{~h}$ e $3 \mathrm{~h}$ ), foram superiores às temperaturas avaliadas antes do estresse. Concluiu-se que após serem submetidos a diferentes tempos de exposição às altas temperaturas, frangos de corte da linhagem $\mathrm{Cobb}^{\circledR}$ com 41 dias de idade aumentaram a temperatura superficial média e cloacal.
\end{abstract}

PALAVRAS-CHAVE: Ambiência animal, aves, estresse cíclico por calor, temperatura corporal, temperatura superficial.

\section{CHARACTERISTICS THERMOREGULATION BEFORE AND AFTER DIFFERENT TIMES OF EXPOSURE TO HEAT IN BROILER}

\begin{abstract}
This study aimed to investigate the effect of heat stress after one, two or three hours in thermoregulatory characteristics of broiler chickens evaluated at 41 days of age.
\end{abstract}


560 broiler chicks were housed, male, of $\mathrm{Cobb} \AA$ lineage, distributed in 16 boxes with 35 birds in each pen. The first to the 13th day of age were raised in ideal room temperature. On the 14th day were created in four thermal environments one in thermal comfort and the other three environments under high ambient temperature by one, two and three hours, respectively. At 41 days of age before and after heat stress were measured the crest, head, wing, back and paw temperatures and cloacal temperature and calculated the average surface temperature (AST). At the time the evaluations were measured air temperatures and the globe, humidity and air velocity. They calculated the mean and standard deviation of thermoregulatory measures for the four thermal environments in the periods before and after heat stress. The unpaired t-test was used to compare the mean of the before group to the after group the heat stress in each thermal environment. The cloacal and surface temperatures after exposure to heat in the three thermal environments (1h, $2 \mathrm{~h}$ and $3 \mathrm{~h})$, were higher than the temperature measured before the stress. It was concluded that after undergoing different times of exposure to high temperatures, broilers Cobb® line with 41 days of age increased the average surface temperature and cloacal.

KEYWORDS: Animal ambience, poultry, cyclic heat stress, body temperature, surface temperature.

\section{INTRODUÇÃO}

A avicultura é uma atividade importante na economia do Brasil, de modo que o país ocupou o primeiro lugar no ranking de exportação de carne de frango $(4,099$ mil toneladas, seguido pelos EUA-3,297 mil ton) e terceiro lugar na produção mundial desse tipo de carne (12,691 milhões de toneladas) em 2014 (ABPA, 2015). O país possui uma enorme diversidade climática, apresentando muitas regiões nas quais imperam temperaturas elevadas. Frente a isso, durante seu crescimento, as aves lançam mão de respostas fisiológicas visando a termorregulação, realizando principalmente o controle da temperatura profunda, mediante vasodilatação ou vasoconstrição periférica (NÄÄS et al., 2010). Devido a relevância da atividade avícola somada aos obstáculos climáticos, a qualidade da produção e o bem-estar animal devem ser garantidos para que a expansão desse setor ocorra de maneira sustentável.

Sabe-se que frangos encontram problemas em relação à transferência de calor para o ambiente quando criados em temperatura ambiente acima do conforto térmico. Consequentemente, pode prejudicar o seu desempenho. Sendo assim, estudar as alterações no metabolismo, nos parâmetros fisiológicos, frente a elevações de temperatura, faz-se necessário, pois com isso o produtor poderá se atentar a essas questões e evitar os efeitos deletérios que o ambiente pode provocar. Assim como NASCIMENTO et al. (2012) elucidaram, muitos estudos definem as alterações fisiológicas dos animais frente ao estresse, contudo, estabelecer uma relação entre exposição de calor e mudanças nas condições dos animais, ainda é necessário.

Dissipação de calor por convecção e por radiação ocorre com mais ênfase quando os animais se encontram em condições termoneutras, comparado a ambientes de temperatura ambiente elevada (NASCIMENTO et al., 2014). Todavia, em condições estressantes, a maior perda de calor se dá pela evaporação respiratória, mediante o aumento na sua frequência. Relataram também que os modelos utilizados para prever a temperatura superficial das aves devem considerar 
as diferenças entre áreas com e sem penas durante todo o período de criação dos animais.

As medidas de temperaturas superficiais dos animais são parâmetros que mudam de maneira rápida de acordo com as condições ambientais; permitem avaliar a condição térmica da ave no ambiente em que ela se encontra e podem ser usadas para mensurar o fluxo sanguíneo periférico e a troca de calor que o animal faz com o ambiente (NASCIMENTO et al., 2012). Há relatos também que há correlação entre temperaturas superficiais e interna dos animais e que, aumentando-se a temperatura ambiente, paralelamente, ocorre o aumento das temperaturas fisiológicas das aves (GILOH et al., 2012).

O mesmo foi observado por NASCIMENTO et al. (2011), que verificaram aumento da temperatura superficial junto ao aumento da temperatura ambiente além de observar especificamente as temperaturas superficiais da asa, cabeça, perna, dorso e crista, observando que o valor que teve maior diferença com a temperatura ambiente foi a temperatura da perna, concordando com NÄÄS et al. (2010), que acrescentam que as partes do corpo que não possuem penas variam mais quando a temperatura ambiente muda, em relação as partes com penas.

Outra observação importante é que animais mais velhos possuem mais penas e devido a isso a temperatura superficial tende a ser mais alta. Além disso, quanto maior o peso vivo das aves, mais sensíveis são a elevadas temperaturas (NASCIMENTO et al., 2014), elucidando a preocupação com a ambiência quando as mesmas estão próximo ao abate.

Dessa forma, objetivou-se investigar o efeito do estresse térmico após uma, duas ou três horas sobre características termorreguladoras avaliadas aos 41 dias de idade em frangos de corte da linhagem $\mathrm{Cobb}^{\circledR}$ criados sob estresse cíclico do $14^{\circ}$ dia até o abate.

\section{MATERIAL E MÉTODOS}

Os procedimentos realizados nesta pesquisa foram aprovados pelo Comitê de Ética na Utilização de Animais (CEUA) da Universidade Federal de Uberlândia, pelo Protocolo Registro CEUA/UFU 065/14.

O estudo foi realizado na Granja de Experimentação de Aves, instalada na Fazenda do Glória, pertencente à Faculdade de Medicina Veterinária da Universidade Federal de Uberlândia, em Uberlândia, Minas Gerais, de abril a maio de 2015. O galpão utilizado é edificado em alvenaria e tem estrutura de metal, coberto com telhas de fibrocimento e forrado com plástico, piso de concreto, paredes com telas, possuindo cortinas laterais duplas (interna e externa), para controle da temperatura e umidade são utilizados ventiladores e nebulizadores; além de campânulas de infravermelhas estas, utilizadas de um a sete dias de idade das aves. A cama das aves era de maravalha.

Foram alojados 560 pintos de corte da linhagem $\mathrm{Cobb}^{\circledR}$, machos, pesando em média $43 \mathrm{~g}$ com um dia de idade, 16 boxes medindo $1,50 \times 1,90 \mathrm{~m}$. Os ovos foram provenientes de matrizes que pertenciam a um mesmo lote, a incubação foi feita numa mesma máquina com condições análogas.

A dieta dos animais ficou restrita a uma ração de níveis nutritivos baseados em ROSTAGNO et al. (2011). Os ingredientes (base sorgo+farelo de soja) que a compuseram tiveram sua matéria prima bromatologicamente testada. A alimentação ocorreu em quatro fases preparadas da seguinte forma: ração pré-inicial, inicial, engorda e abate, atendendo a exigências energética e nutricional. Junto à ração os 
animais receberam água potável à vontade com um programa luz distribuído da seguinte maneira: um a sete dias: duas horas sem luz; oito a 21 dias, quatro horas sem luz; 22 a 42 dias, duas horas sem luz.

Do primeiro ao $13^{\circ}$ dia de idade as aves foram mantidas em condições térmicas de acordo com o manual de criação de frangos de corte da linhagem $\mathrm{Cobb}^{\circledR}$ (2008). A partir do $14^{\circ}$ dia, o aviário foi separado em quatro câmaras paralelas de $5,60 \times 10,20 \times 2,8 m$ delimitadas por cortinas plásticas dupla face ao longo da largura do galpão, dobradas de modo a expor a face branca. Assim, obtiveram-se quatro ambientes térmicos distintos compostos por quatro boxes cada. No ambiente controle, as aves foram criadas conforme recomendação térmica para a linhagem. Nos demais ambientes, as aves foram submetidas ao estresse por calor, respectivamente, por $1 \mathrm{~h}, 2 \mathrm{~h}$ e $3 \mathrm{~h}$ diárias, com início às 11:00h.

O estresse cíclico pelo calor foi gerado por meio de campânulas de infravermelho com temperatura de $8^{\circ} \mathrm{C}$ acima da recomendada para a idade das aves. Simultaneamente ao aquecimento artificial, os ventiladores permaneceram ligados. Os valores de $\mathrm{CO}_{2}$ não ultrapassaram o recomendado (<3000ppm).

Aos 41 dias de idade, quatro aves escolhidas ao acaso em cada boxe tiveram as temperaturas da superfície corporal e da cloaca avaliadas, totalizando 16 animais por ambiente térmico, as quais foram denominadas como medidas ANTES. Após o estresse de $1 \mathrm{~h}, 2 \mathrm{~h}$ e $3 \mathrm{~h}$ esse procedimento foi novamente realizado e essas avaliações nomeadas como medidas DEPOIS. As medidas DEPOIS das aves do ambiente controle referem-se à mensuração realizada três horas após a medida ANTES, coincidindo com o final do período de estresse do ambiente com 3 horas de exposição ao calor.

A temperatura superficial foi quantificada com termômetro digital infravermelho Instrutemp DT8530 nas regiões da crista, cabeça, asa, dorso e canela. Já a temperatura cloacal foi medida com termômetro de mercúrio Incoterm L015/13 inserido a $3 \mathrm{~cm}$ na cloaca por dois minutos.

Calculou-se a temperatura superficial média (TSM) utilizando a recente equação proposta por NASCIMENTO (2010) que leva em consideração linhagem e a idade das aves em semanas, ponderando as diferentes regiões corporais avaliadas:

$$
\text { TSM }=0,27^{\star} \text { Tasa }+0,10^{\star} \text { Tcabeça }+0,05^{\star} \text { Tpata }+0,50^{\star} \text { Tdorso }+0,10^{\star} \text { Tcrista }
$$

em que:

Tasa $=$ temperatura da asa; Tcabeça $=$ temperatura da cabeça; Tpata $=$ temperatura da pata; Tdorso = temperatura do dorso e Tcrista = temperatura da crista.

Para caracterização do ambiente térmico, antes e após estresse aos 41 dias de idade, foram coletadas as temperaturas de bulbo seco, bulbo úmido e globo negro por meio do termômetro globo modelo IBUTG de marca $\mathrm{Homis}^{\circledR}$ e a velocidade do vento por anenômetro digital (marca Instrutherm modelo AD-250) (Tabela 1).

Foram calculados a média e o desvio-padrão das medidas de termorregulação para os quatro ambientes térmicos nos períodos antes e após os três períodos de estresse por meio do procedimento PROC MEANS do pacote estatístico $S A S^{\circledast}$. O teste t não-pareado foi utilizado para comparar as médias dos grupos antes e depois do estresse por calor em cada ambiente térmico. 
TABELA 1: Médias e desvios padrão da temperatura, umidade relativa, ITU, ITE e ITGU antes e depois da exposição ao estresse por calor.

\begin{tabular}{|c|c|c|c|c|}
\hline \multicolumn{5}{|c|}{ Ambiente térmico (antes) } \\
\hline & Controle (Oh) & $1 \mathrm{~h}$ & $2 \mathrm{~h}$ & $3 \mathrm{~h}$ \\
\hline $\mathrm{T}\left({ }^{\circ} \mathrm{C}\right)$ & $27,0 \pm 1,2$ & $26,0 \pm 0,2$ & $27,1 \pm 0,2$ & $27,2 \pm 0,0$ \\
\hline UR (\%) & $72 \pm 8,0$ & $66 \pm 0,6$ & $84 \pm 0,6$ & $84 \pm 0,0$ \\
\hline ITU & $76 \pm 0,5$ & $74 \pm 0,3$ & $78 \pm 0,3$ & $78 \pm 0,0$ \\
\hline ITE & $31 \pm 0,4$ & $29 \pm 0,3$ & $34 \pm 0,4$ & $34 \pm 0,0$ \\
\hline ITGU & $80 \pm 2,1$ & $79 \pm 0,3$ & $80 \pm 0,3$ & $80 \pm 0,0$ \\
\hline $\mathrm{VV}\left(\mathrm{m} \cdot \mathrm{s}^{-1}\right)$ & $0,6 \pm 0,6$ & $0,0 \pm 0,0$ & $0,0 \pm 0,0$ & $0,0 \pm 0,0$ \\
\hline \multicolumn{5}{|c|}{ Ambiente térmico (depois) } \\
\hline & Controle (Oh) & $1 \mathrm{~h}$ & $2 \mathrm{~h}$ & $3 \mathrm{~h}$ \\
\hline $\mathrm{T}\left({ }^{\circ} \mathrm{C}\right)$ & $27,6 \pm 0,0$ & $30,3 \pm 0,1$ & $30,4 \pm 0,1$ & $30,2 \pm 0,2$ \\
\hline UR (\%) & $55 \pm 0,0$ & $81 \pm 0,0$ & $79 \pm 2,2$ & $81 \pm 1,7$ \\
\hline ITU & $74 \pm 0,0$ & $82 \pm 0,1$ & $81 \pm 0,1$ & $80 \pm 0,0$ \\
\hline ITE & $29 \pm 0,0$ & $39 \pm 0,1$ & $38 \pm 0,3$ & $36 \pm 0,0$ \\
\hline ITGU & $82 \pm 0,0$ & $85 \pm 0,2$ & $85 \pm 0,2$ & $83 \pm 0,3$ \\
\hline $\mathrm{VV}\left(\mathrm{m} \cdot \mathrm{s}^{-1}\right)$ & $0,4 \pm 0,0$ & $0,1 \pm 0,0$ & $0,0 \pm 0,0$ & $0,0 \pm 0,0$ \\
\hline
\end{tabular}

T: temperatura; UR: umidade relativa; ITU: Índice de Temperatura e Umidade; ITE: Índice de Temperatura Equivalente; ITGU: Índice de Temperatura de Globo Negro e Umidade; VV: velocidade do vento.

\section{RESULTADOS E DISCUSSÃO}

As temperaturas cloacal e superficiais, após exposição ao calor nos três ambientes térmicos ( 1,2 e $3 \mathrm{~h})$, foram superiores às temperaturas avaliadas antes do estresse, exceto a temperatura da cabeça que permaneceu constante quando as aves foram expostas a 2 e 3 horas de calor (Tabela 2).

A temperatura da cloaca indica a temperatura do centro corporal (ou seja, interna) e pode valer-se como um parâmetro de avaliação para saber se a ave está em conforto ou estresse (BROWN-BRANDTL et al., 2003). Em condições naturais de temperatura e umidade, a temperatura da cloaca de frangos de corte machos aos 42 dias é de aproximadamente $41^{\circ} \mathrm{C}$ (NASCIMENTO et al., 2012; BUENO et al., 2014).

NASCIMENTO et al. (2012) submetendo frangos de corte ao estresse pelo calor em diferentes tempos de exposição observaram na sexta semana (36-42dias) um aumento da temperatura cloacal após o estresse somente nas aves que ficaram expostas ao calor por mais de uma hora, não se alterando em períodos menores de exposição ao calor. Estudando frangos de corte em temperaturas superiores as deste estudo $\left(38-40^{\circ} \mathrm{C}\right)$ durante uma hora por dia MARCHINI et al. (2007) observaram aos 42 dias de idade temperatura cloacal de $41,2^{\circ} \mathrm{C}$ antes do estresse e de $42,7^{\circ} \mathrm{C}$ após o estresse de calor, concluindo que em uma hora expostos a altas temperaturas, os frangos de corte aumentam a temperatura cloacal, concordando com os resultados desta pesquisa. 
TABELA 2: Médias e desvios padrão das temperaturas cloacal e superficial, em ${ }^{\circ} \mathrm{C}$, de frangos de corte da linhagem $\mathrm{Cobb}{ }^{\circledR}$ antes e após exposição ao estresse por calor.

Tempo de exposição ao estresse

\begin{tabular}{|c|c|c|c|c|c|c|c|c|}
\hline \multicolumn{3}{|c|}{ Controle } & \multicolumn{2}{|c|}{$1 \mathrm{~h}$} & \multicolumn{2}{|c|}{$2 \mathrm{~h}$} & \multicolumn{2}{|c|}{$3 \mathrm{~h}$} \\
\hline & Antes & Depois & Antes & Depois & Antes & Depois & Antes & Depois \\
\hline $\mathrm{TC}$ & $41,9 \pm 0,2$ & $41,7 \pm 0,3$ & $41,8 \pm 0,1 a$ & $42,4 \pm 0,4 b$ & $41,8 \pm 0,2 a$ & $42,3 \pm 0,1 b$ & $41,6 \pm 0,1 a$ & $42,3 \pm 0,5 b$ \\
\hline Tdorso & $32,5 \pm 0,9$ & $32,3 \pm 0,8$ & $32,7 \pm 0,7 a$ & $35,2 \pm 1,1 b$ & $32,7 \pm 0,7 a$ & $35,4 \pm 1,1 b$ & $32,8 \pm 0,9 a$ & $35,7 \pm 0,7 b$ \\
\hline Tcrista & $33,4 \pm 0,2$ & $35,3 \pm 1,7$ & $34,9 \pm 0,8 a$ & $37,5 \pm 1,0 b$ & $35,1 \pm 1,0 a$ & $37,6 \pm 1,5 b$ & $33,5 \pm 0,8 a$ & $37,7 \pm 2,1 b$ \\
\hline Tcabeça & $33,6 \pm 2,1$ & $32,1 \pm 1,2$ & $32,5 \pm 0,8 a$ & $35,5 \pm 0,7 b$ & $34,3 \pm 1,2 a$ & $35,3 \pm 0,7 a$ & $35,7 \pm 1,0 a$ & $35,6 \pm 0,3 a$ \\
\hline
\end{tabular}

TC: temperatura cloacal; Tasa: temperatura da asa; Tdorso: temperatura do dorso; Tcrista: temperatura da crista; Tcabeça: temperatura da cabeça; Tpata: temperatura da pata e TSM: temperatura superficial média.

Dentro de cada tempo de exposição ao estresse pelo calor, médias seguidas por letras diferentes na linha diferem pelo teste "t" a $5 \%$. 
MARCHINI et al. (2007) justificam o aumento da temperatura cloacal nas aves, em ambientes com altas temperaturas, pela ineficiência na dissipação de calor sensível devido à pequena diferença entre a temperatura ambiente e da pele. $A$ elevação exorbitante da temperatura cloacal em situações de estresse térmico pode elevar a mortalidade de frangos de corte (SILVA et al., 2007) principalmente das linhagens de rápido crescimento, o que é uma preocupação para o sistema produtivo.

NASCIMENTO et al. (2011) observaram que a média da temperatura superficial das regiões cobertas com pena é menor $\left(30,8^{\circ} \mathrm{C}\right)$ que das regiões sem penas $\left(37,9^{\circ} \mathrm{C}\right)$, em temperaturas ambientes com média de $28^{\circ} \mathrm{C}$ e máxima e mínima de $34,3^{\circ} \mathrm{C}$ e $18^{\circ} \mathrm{C}$, respectivamente. Esse mesmo comportamento foi observado no presente estudo, independente do tempo de exposição ao calor que aves foram submetidas, exceto para a temperatura da cabeça com 2 e $3 \mathrm{~h}$ de exposição.

O aumento da temperatura superficial após o estresse térmico observado no presente estudo é justificado por sua alta correlação com a temperatura ambiental. Entretanto, de acordo com NÄÄS et al. (2010) existe uma maior correlação entre as áreas sem penas (crista e pata) e a temperatura ambiente, já que estas regiões respondem mais rápido as alterações no ambiente do galpão em relação as regiões com penas (cabeça, dorso e asa). Em seus estudos as médias das temperaturas de crista e pata foram de $38,8^{\circ} \mathrm{C}$ e $39,4^{\circ} \mathrm{C}$ respectivamente; enquanto da asa, cabeça e dorso foram de $32,5^{\circ} \mathrm{C}$; $34^{\circ} \mathrm{C}$ e $32,8^{\circ} \mathrm{C}$; mensuradas em frangos da mesma linhagem e idade do presente estudo e submetidas a temperaturas médias de $29,3 \pm 2,0^{\circ} \mathrm{C}$.

De acordo com NASCIMENTO et al. (2014), independente da idade em que se avalie a temperatura superficial de frangos de corte deve-se levar em conta 0 quanto todas as superfícies (exceto a crista, ainda em formação) contribuem para o cálculo da temperatura superficial média (TSM), pois mesmo que as aves ainda apresentem seu sistema termorregulador imaturo e penas em formação, ainda assim, existe uma distinção entre a temperatura das áreas com e sem penas.

A temperatura superficial média (TSM) também aumentou após o estresse em todos os ambientes em que as aves foram expostas ao calor. Nos ambientes de exposição ao calor por $2 \mathrm{~h}$ e $3 \mathrm{~h}$, a TSM ultrapassou o intervalo proposto por SCHÜTZ (2011) que em condições naturais de temperatura e umidade varia de 32,9 a $34,9^{\circ} \mathrm{C}$ aos 41 dias de idade em frangos de corte. Isso ressalta a importância de se considerar não só o tipo de estresse (crônico, agudo, cíclico ou constante) e a temperatura que as aves foram submetidas, mas também o tempo de exposição.

GILOH et al. (2012) constataram que o aumento da temperatura ambiente culminou no acréscimo da temperatura superficial das aves, devido a vasodilatação periférica, a qual é uma resposta fisiológica para aumentar a dissipação térmica.

\section{CONCLUSÃO}

Concluiu-se que após serem submetidos a diferentes tempos de exposição a altas temperaturas, frangos de corte da linhagem Cobb ${ }^{\circledR}$ com 41 dias de idade aumentaram a temperatura superficial média e cloacal.

\section{AGRADECIMENTOS}

A FAPEMIG (Fundação de Amparo à Pesquisa do Estado de Minas Gerais) pelo financiamento da pesquisa (Universal APQ-01292-13). 


\section{REFERÊNCIAS}

ABPA. Associação Brasileira de Proteína Animal- ABPA. São Paulo, SP, 2015. Disponível em: <www.ubabef.com.br>. Acesso em: 02 set. 2015.

BROWN-BRANDL, T. M.; YANAGI JÚNIOR, T.; XIN, H.; GATES, R. S.; BUCKLIN, R. A. A new telemetry system for measuring core body temperature in livestock and poultry. Applied Engineering in Agriculture, St. Joseph, v. 19, n. 5, p. 583-589, 2003.

BUENO, J. P. R.; NASCIMENTO, M. R. B. M.; CARVALHO, C. M. C.; SILVA, M. C. A.; SILVA, P. L. A. P. A. Características de termorregulação em frangos de corte, machos e fêmeas, criados em condições naturais de temperatura e umidade. Enciclopédia Biosfera: Centro Científico Conhecer, Goiânia, v. 10, n. 19, p. 437447, 2014.

COBB-VANTRESS BRASIL. Manual de manejo de frangos de corte. Guapiaçu: Cobb Vantress, 2008. 66p.

GILOH, M.; SHINDER, D.; YAHAV, S. Skin surface temperature of broiler chickens is correlated to body core temperature and is indicative of their thermoregulatory status. Poultry Science, Champaign, v. 91, n. 1, p. 175-188, 2012.

MARCHINI, C. F. P.; SILVA, P. L.; NASCIMENTO, M. R. B. M.; TAVARES, M. Frequência respiratória e temperatura cloacal em frangos de corte submetidos à temperatura ambiente cíclica elevada. Archives of Veterinary Science, Curitiba, v. 12, n. 1, p. 41-46, 2007.

NÄÄS, I. A. ROMANINI, C. E. B.; NEVES, D. P.; NASCIMENTO, G. R.; VERCELLINO, R. A. Broiler surface temperature distribution of 42 day old chickens. Scientia Agricola, Piracicaba, v. 67, n. 5, p. 497-502, 2010.

NASCIMENTO, S. T. Determinação do balanço de calor em frangos de corte por meio das temperaturas corporais. 2010. Dissertação (Mestrado em Ciências) Escola Superior de Agricultura "Luiz de Queiroz", Universidade de São Paulo. $147 f$.

NASCIMENTO, G. R.; PEREIRA, D. F.; NÄÄS, I. A.; RODRIGUES, L. H. A. Índice Fuzzy de conforto térmico para frangos de corte. Engenharia Agrícola, Jaboticabal, v. 31, n. 2, p. 219-229, 2011.

NASCIMENTO, S. T.; SILVA, I. J. O.; MOURÃO, G. B.; CASTRO, A. C. Bands of respiratory rate and cloacal temperature for different broiler chicken strains. Revista Brasileira de Zootecnia, Viçosa, v. 41, n. 5, p. 1318-1324, 2012.

NASCIMENTO, S. T.; SILVA, I. J. O.; MAIA, A. S. C.; CASTRO, A. C.; VIEIRA, F.M. C. Mean surface temperature prediction models for broiler chickens - a study of sensible heat flow. International Journal of Biometeorology, Lisse, v. 58, n. 2, p. 195-201, 2014. 
ROSTAGNO, H. S.; ALBINO, L. F. T.; DONZELE, J. L.; GOMES, P. C.; OLIVEIRA, R. F.; LOPES, D. C.; FERREIRA, A. S.; BARRETO, S. L. T.; EUCLIDES, R. F. Tabelas brasileiras para aves e suínos: composição de alimentos e exigências nutricionais. Viçosa: UFV-DZO, 2011. 252p.

SHÜTZ, E. S. Variabilidade do ambiente térmico em galpão para frangos de corte e sua influência nas respostas fisiológicas e comportamento das aves. 2011. Programa de Pós-Graduação em Ciência Animal, Universidade Federal de Goiás. 88f.

SILVA, M. A. N.; BARBOSA FILHO, J. A. D.; SILVA, C. J. M.; ROSÁRIO, M. F.; SILVA, I. J. O.; COELHO, A. A. D.; SAVINO, J. M. Avaliação do estresse térmico em condição simulada de transporte de frangos de corte. Revista Brasileira de Zootecnia, Viçosa, v.36, n.4, p.1126 -1130, 2007 (supl.). 